\begin{tabular}{|l|l} 
REVISTA & $\begin{array}{l}\text { Revista Educación } \\
\text { ISSN: 0379-7082 } \\
\text { ISSN: 2215-2644 } \\
\text { revedu@ gmail.com } \\
\text { Universidad de Costa Rica } \\
\text { Costa Rica }\end{array}$
\end{tabular}

\title{
Edadismo en la escuela. ¿Tienen estereotipos sobre la vejez los futuros docentes?
}

Gutiérrez Moret, Margarita; Mayordomo Rodríguez, Teresa

Edadismo en la escuela. ¿Tienen estereotipos sobre la vejez los futuros docentes?

Revista Educación, vol. 43, núm. 2, 2019

Universidad de Costa Rica, Costa Rica

Disponible en: http://www.redalyc.org/articulo.oa?id=44058158029

DOI: https://doi.org/10.15517/revedu.v43i2.32951

Esta obra está bajo una Licencia Creative Commons Atribución-NoComercial-SinDerivar 3.0 Internacional. 


\section{Edadismo en la escuela. ¿'Tienen estereotipos sobre la vejez los futuros docentes?}

Ageism in the School: Do Stereotypes about Ageing Exist among Future Teachers?

Margarita Gutiérrez Moret

Universidad Católica de Valencia, España

margarita.gutierrez@ucv.es

(iD) http://orcid.org/0000-0001-5247-1777

Teresa Mayordomo Rodríguez

Universidad Católica de Valencia, España

teresa.mayordomo@ucv.es

iD http://orcid.org/orcid 0000-0003-1873-4910
DOI: https://doi.org/10.15517/revedu.v43i2.32951

Redalyc: http://www.redalyc.org/articulo.oa?id=44058158029

Recepción: 12 Septiembre 2018

Aprobación: 05 Junio 2019

\section{Resumen:}

En este artículo se presentan los datos de un estudio piloto descriptivo en el cual se han medido los estereotipos sobre la vejez en una muestra de carácter incidental de estudiantado universitario del grado de maestro de Educación Primaria. El objetivo fue analizar si hay estereotipos sobre la vejez a través del Cuestionario de Evaluación de Estereotipos Negativos sobre la Vejez [CENVE] (Blanca, Sánchez y Trianes, 2005) en 78 estudiantes. Los resultados mostraron la presencia de estereotipos que plantearon la reflexión sobre la necesidad de proponer actividades educativas que contribuyan a mejorar la imagen de las personas mayores, tanto en la formación de los docentes como en la de los niños en la etapa de la educación primaria.

Palabras Clave: envejecimiento, estereotipos, educación, docentes, vejez.

\section{AbSTRACT:}

This article shows the results of a descriptive pilot study that measures stereotypes regarding ageing. The incidental sample of 78 students was drawn from a group of college students majoring in Primary School Education. The primary goal was to determine the existence of stereotypes associated with ageing using the CENVE questionnaire (Questionnaire on the Evaluation of Negative Stereotypes on Ageing, (Blanca, Sánchez y Trianes, 2005). The results revealed that stereotypes do, in fact, exist and that more educational outreach is required for both teachers and primary school students to dismiss negative stereotypes about the elderly.

KEYWORDS: Ageing, Stereotypes, Education, Teachers, elderly.

\section{INTRODUCCIÓN}

En los últimos años ha tomado fuerza el estudio de las ideas y teorías implícitas del cuerpo docente y el análisis de la influencia que tienen estas creencias en su labor educativa (López y Basto, 2010; Medina, 2014).

Aunque el trabajo del profesorado de educación primaria se desarrolla con niños, sus creencias sobre la vejez y las personas de edad avanzada tienen un impacto en la educación de sus alumnos a través de la transmisión de valores y cultura.

La sociedad tiene una imagen de las personas mayores y de la vejez basada en una serie de estereotipos que suelen ser falsos y que influyen sobre las actitudes y comportamientos de todos los que interactúan con personas mayores.

Este trabajo pretende acercarse a las creencias de futuros profesores a través del análisis de los datos obtenidos en el cuestionario de evaluación de estereotipos negativos sobre la vejez y reflexionando a su vez sobre la imagen de las personas mayores en el ámbito educativo. 


\section{MARCO TEÓRICO}

La vejez se ha valorado de dos formas, una positiva y otra negativa. La primera, se refiere a la consideración de la persona mayor como sabia, cargada de experiencias, de alto estatus social, merecedora de un gran respeto y con una clara posición de influencia sobre los demás. La segunda, destaca la vejez como un estado deficitario; la edad lleva consigo pérdidas significativas e irreversibles (Carbajo, 2009). Es de señalar que, se ha pasado de un modelo de déficit intelectual y encasillado en la demencia a un modelo el cual reconoce el potencial de aprendizaje de las personas a lo largo de todo el ciclo vital, la plasticidad y la capacidad de compensación y optimización ante los cambios (Baltes, Staudinger y Lindenberger, 1999).

El modelo negativo del envejecimiento ha sido el dominante no solo desde las ciencias médico-biológicas, sino también desde las ciencias sociales y del comportamiento. También las representaciones sociales juegan un papel importante en el modelo negativo de vejez, el cual a pesar de todos los cambios, todavía permanece en nuestra sociedad en forma de prejuicios y estereotipos.

A pesar de que el envejecimiento supone uno de los grandes logros de los avances de la ciencia en nuestra sociedad, la imagen de las personas mayores parece que no goza del reconocimiento que este hecho significa. De acuerdo con Reyes, Altamar, Aguirre y Murillo, (2014) explorar los factores culturales y generacionales que influyen en los significados de la vejez y las personas mayores influyen en el bienestar de este grupo.

El interés en el estudio de los estereotipos reside en sus implicaciones prácticas, pues las creencias sobre la vejez pueden traducirse en conductas discriminatorias hacia las personas mayores. Este proceso de discriminación fue acuñado con el término ageism en referencia a estereotipos o actitudes prejuiciosas mantenidas hacia una persona solo por el hecho de ser una persona mayor (Butler, 1969, Palmore, 1990). Este concepto, se ha traducido al castellano como edadismo (Montorio, Izal, Sánchez y Losada, 2002).

Los estereotipos sobre la vejez afectan a diferentes colectivos: jóvenes y adultos, familiares de los mayores, profesionales de la salud, e incluso a la propia persona mayor (Fuentes, 2014; Bustillos y Fernández, 2013; León, Correa y Giacaman, 2015).

La psicología ha constatado que la imagen que tenemos de las personas mayores se proyecta en nuestra conducta. Los estereotipos activan comportamientos determinados, como ocurre en la profecía autocumplida. En este caso, durante las interacciones sociales con personas mayores, las expectativas de otros grupos de edad sirven de guía a su conducta, anticipándose al comportamiento de la persona mayor y organizando su respuesta. De la misma forma, la conducta del mayor trata de ajustarse a lo esperado por el otro, lo que tiende a confirmar el estereotipo.

Palmore (1990), considera que los estereotipos proporcionan una imagen exagerada de pocas características, que tienden a valorarse como válidas y verdaderas porque se relacionan con conductas que pueden tener una parte de verdad. Por ejemplo, uno de los estereotipos más extendidos es el de que las personas mayores constituyen un grupo homogéneo. Sin embargo, la ciencia muestra que la heterogeneidad y diversidad es mayor que en otros grupos de edad. Se puede decir que la vejez es una etapa en la cual la variabilidad interindividual alcanza su punto máximo (Vega y Bueno, 1996; Portal de Mayores, 2012). Siempre es posible encontrar alguna persona a quien se le pueda aplicar cualquiera de las creencias erróneas que existen sobre el grupo de personas mayores. Con lo que, siempre encontraremos algún caso que confirme el estereotipo por equivocada que sea.

Cada vez niños y adolescentes conviven más con personas mayores porque la longevidad y esperanza de vida ha aumentado en los últimos años (Abellan y Pujol, 2016). Además, los niños se ven influidos por los estereotipos negativos que se extienden culturalmente sobre las personas mayores.

La educación en el respeto y en una imagen positiva de la vejez es una contribución importante que debe trabajarse en las escuelas. Las personas adultas mayores se han convertido en figura relevante e importante para la educación de los niños y se encuentran cada vez más implicadas en ella. Su presencia en las familias 
de hoy es un elemento insustituible en el proceso de formación de los hijos y las hijas. Pueden y de hecho transmiten valores familiares y mantienen el vínculo entre generaciones (Sanz, Mula y Moril, 2011).

La idea que tienen los niños sobre las personas mayores y el envejecimiento se debe a un conocimiento que ha sido adquirido en el proceso de socialización tanto familiar como escolar y extraescolar (Santamarina y Marinas, 2009). Los niños todavía no han formado estereotipos globales de las personas mayores, solo tienen imágenes estrechamente relacionadas con las experiencias subjetivas de sus familiares. Aunque como señalan, Scholl y Sabat (2008) y Pérez (2004) las influencias de la televisión y otros medios de comunicación cada vez tienen mayor influencia en la formación de ideas sobre la vejez.

El término que suelen utilizar los niños y las niñas para referirse a este colectivo es el de abuelos/as. De ahí la importancia de la proyección de las propias creencias que hacen los educadores en el proceso de socialización de los alumnos, pues esta socialización va más allá de la experiencia subjetiva del niño con sus abuelos y puede repercutir en la visión que menores tienen de las personas mayores.

El Observatorio de Personas Mayores (2009) ejecutó en España una investigación cualitativa para conocer cómo perciben y valoran las personas menores y adolescentes al colectivo de personas mayores. Analizó la evolución de la imagen que tienen los niños en función de la edad. A partir de grupos de discusión estudiaron el discurso emergente mediante las ideas espontaneas que tienen los niños y las niñas sobre las personas mayores. Encontraron que entre los 8 y 10 años, los menores perciben a las personas mayores como una prolongación de los padres; entre los 10 y 12 años como personas autónomas y entre los 11 y 14 años, como colegas. Esta nueva relación, fruto de los cambios sociales, del aumento de la esperanza de vida, de las mejores condiciones de salud de la cual gozan los mayores de hoy, repercute de manera especial en la estructura familiar.

En otro estudio efectuado en España sobre la imagen de los mayores en la infancia, se encontró que niños de ocho años ya tenían presente la cercanía de la muerte en las explicaciones que daban acerca de la salud de las personas mayores (Santamarina, López de Miguel, López y Mendiguren, 2002). La evolución del miedo a la muerte en los niños va ligada a su desarrollo cognitivo. Los niños en la etapa infantil pueden tener miedo a la muerte de sus seres queridos, y relacionar el envejecimiento con la muerte por experiencias vividas o por las respuestas que se les ha dado desde el entorno familiar (Méndez, Inglés, Hidalgo, García y Quiles, 2003). El miedo en el niño está relacionado con el desamparo y ausencia de cuidados, no con el miedo a la muerte en sí misma.

Estos miedos evolucionan en la etapa de la educación primaria hacia la adolescencia y están influenciados por factores de tipo cultural. De hecho, Palmore (1990) señala que la existencia del edadismo parece estar relacionada con el miedo a la muerte, y con la asociación existente entre el envejecimiento, la enfermedad y la muerte, que en nuestra cultura todavía son percibidos como sinónimos.

La visión de la vejez y de las personas mayores para los docentes no solo tiene implicaciones prácticas en cuanto al proceso de socialización y transmisión de valores desde la escuela y en la relación maestro-alumno. Las generaciones de jóvenes docentes también están conviviendo y van a convivir con otros docentes a quienes pueden percibir como pertenecientes al grupo de personas mayores. Esta realidad intergeneracional en el desempeño laboral en la educación conlleva convivir con diferentes metodologías de enseñanza, cambios culturales y formación diversa, por ejemplo, en cuanto a nuevas tecnologías.

El Informe 2014 sobre el estado del sistema educativo destaca la edad de los profesores en España, algo que no parece afectar únicamente a nuestro país. Esta situación nos hace pensar en la convivencia dentro de los centros educativos de docentes pertenecientes a generaciones distintas. Madrid (2005) analizó las percepciones de los profesores y los alumnos en relación con la influencia de la edad del profesorado en la metodología de enseñanza que empleaban y en el rendimiento que consigue en el alumnado, encontrando que se valoraba positivamente la experiencia docente de los profesores de más edad y el entusiasmo de los más jóvenes. 
Aunque hay pocos estudios en esta dirección en nuestro país, otros trabajos internacionales han encontrado aspectos negativos relacionados con la imagen del maestro mayor, Hsu (2007) indica que la edad de jubilación de los docentes en Taiwán genera actitudes edadistas por parte de los docentes jóvenes, de hecho, existe evidencia en términos judiciales sobre esta discriminación por edad hacia los trabajadores mayores (Posthuma y Campion, 2009). Otro de los cambios que se han producido en educación y que influyen en la imagen del profesor mayor, se relaciona con la velocidad de los avances en tecnología aplicada a la educación, donde podemos observar la existencia de una brecha tecnológica entre los profesores jóvenes y mayores en la que las competencias técnicas y los conocimientos en recursos tecnológicos de los profesores disminuyen en cuanto se incrementa la edad (Almerych et ál., 2005).

Además, la discriminación hacia las personas mayores dentro de contextos laborales produce una disminución del rendimiento laboral en las propias personas mayores, generando sentimientos de autoeficacia negativos y repercutiendo en directamente en su salud (Levy, Hausdorff, Henke y Wei, 2000; Levy, Ashman y Dor, 2000).

Las personas mayores son una realidad demográfica y social que está presente en la propia acción educativa, por ello, la importancia de estudiar los estereotipos negativos sobre la vejez de un grupo de futuros maestros de educación primaria. El objetivo de este artículo es analizar qué estereotipos tienen los estudiantes. Este conocimiento supone un primer paso para el diseño de intervenciones educativas en la formación inicial de los maestros que ayuden a combatir los falsos mitos que existen alrededor de la vejez y de las personas mayores.

Los resultados que se presentan en este artículo forman parte de un proyecto más amplio de la Universidad Católica de Valencia con el fin de diseñar actividades formativas para mejorar el conocimiento gerontológico de estudiantes universitarios de diferentes especialidades.

\section{Método}

La muestra del estudio, de carácter incidental está compuesta por 78 estudiantes de la Facultad de Magisterio y Ciencias de la Educación, todos del segundo curso del Grado de Maestro en Educación Primaria. La distribución por sexos es del 34,6\% hombres, y el 65,4\% mujeres. En cuanto a la media de edad es de 20,27 años $(\mathrm{DT}=2,93)$.

Para la elaboración de este estudio descriptivo y transversal, todo el alumnado fue informado sobre la investigación y quienes dieron su consentimiento para participar respondieron al cuestionario, teniendo una duración aproximada de 20 minutos. Se siguieron todos los procedimientos establecidos para la investigación en la Facultad de Magisterio y Ciencias de la Educación de la Universidad Católica de Valencia.

El instrumento utilizado para medir los estereotipos negativos hacia las personas mayores es el Cuestionario de Evaluación de Estereotipos Negativos sobre la Vejez (CENVE) (Blanca et ál., 2005), que supone una adaptación del cuestionario Facts on Aging Quiz (FAQMH) de Palmore (1988). La escala utilizada consta de un total de 15 ítems con formato de respuesta tipo Likert ( $1=$ muy en desacuerdo, $4=$ muy de acuerdo). Sus autoras recomendaban calcular tres subescalas sobre estereotipos negativos relacionados con la salud (que mide las creencias sobre el deterioro de memoria, enfermedades mentales, fuerte deterioro de la salud, incapacidades por las que dependen de los demás y el deterioro cognitivo como parte inevitable de la vejez), el ámbito motivacional-social (los ítems se refieren a tener menos interés por el sexo, tener menos amigos, pérdida de interés por las cosas, ser incapaces de resolver los problemas, no ejecutar un trabajo tan bien como los más jóvenes), y el carácter y la personalidad (los ítems hacen referencia a que los mayores se irritan y son cascarrabias, se vuelven más rígidas e inflexibles, son como niños, los defectos de la gente se agudizan con la edad, Chochean).

Las puntuaciones de las dimensiones oscilan entre 5 y 20, y según Franco, Villarreal, Martínez y Galicia (2010) cuando la puntuación se identifica entre 12,5 y 20 denotan un alto grado de estereotipo negativo sobre la vejez en esa dimensión. El alfa de Crombach para la escala total es de, 826. En la Tabla 1 pueden observarse 
las puntuaciones medias y desviaciones típicas obtenidas para cada una de esas subescalas que componen el test y para el test total.

TABLA 1

Puntuaciones medias para el CENVE y sus escalas

\begin{tabular}{lll}
\hline & Media & $\begin{array}{l}\text { Desviación } \\
\text { tipica }\end{array}$ \\
\hline CENVE total & 38,83 & 6,77 \\
Salud & 12,39 & 2,36 \\
Motivación & 13,05 & 2,82 \\
Social & & \\
Personalidad & 13,38 & 2,73 \\
\hline
\end{tabular}

Fuente: Elaboración propia

\section{Resultados}

Del estudiantado de magisterio que ha sido evaluado, un 43,6\% no muestra estereotipos negativos en sus respuestas al CENVE, mientras que $56,4 \%$ sí que presentan estereotipos negativos sobre la vejez. Concretamente, si se efectúa un análisis más profundo del porcentaje de hombres y mujeres que tienen o no estereotipos negativos hacia la vejez, se observa que de ese $56,4 \%$ de estudiantes que muestran estereotipos negativos hacia la vejez, el $68,18 \%$ son mujeres, lo que podría indicarnos que el género femenino posee mayor estereotipo hacia la vejez que los hombres. En la Figura 1 puede verse la representación de hombres y mujeres con estereotipos sobre la vejez.

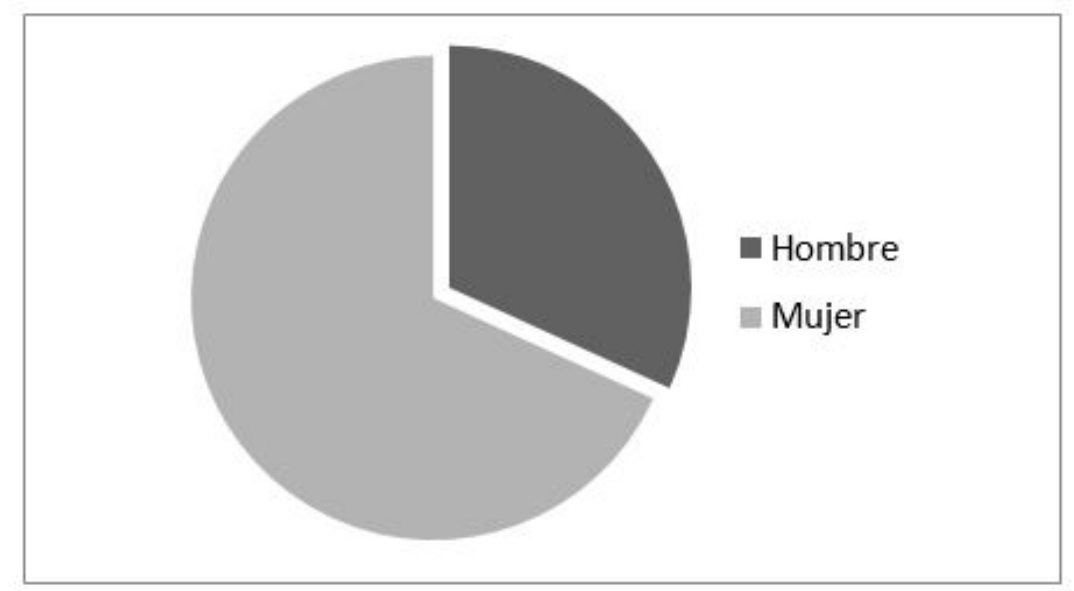

FIGURA 1

Porcentaje de hombres y mujeres con estereotipos negativos hacia la vejez Fuente: Elaboración propia

Por lo que respecta al grupo, la media de estereotipos es de 38,83 (DT=6,77), lo que señala que existen estereotipos negativos sobre la vejez.

Si se indaga un poco más y se analiza las diferencias entre hombres y mujeres tanto en CENVE total $(\mathrm{t}$ $(76)=-, 017=\mathrm{p}=, 986$ ) como en las subescalas que lo componen (salud, $\mathrm{t}(76)=-, 273=\mathrm{p}=, 785$; motivación social, $\mathrm{t}(76)=-, 032=\mathrm{p}=, 974$; personalidad, $\mathrm{t}(76)=, 226=\mathrm{p}=, 822)$ se observa que no existen diferencias significativas entre ellos. En la Figura 2 pueden notarse las puntuaciones obtenidas para cada una de las subescalas. Tal y como puede verse, la única dimensión en la que no se presentan estereotipos negativos es 
salud, en la que la media de hombres es de $12,29(\mathrm{DT}=2,14)$ y la de mujeres 12,45 (DT=2,49), no obstante, puede verse cómo es en el grupo de mujeres donde las puntuaciones son más altas. Por lo que respecta a las puntuaciones totales la media para hombres de $38,81(\mathrm{DT}=5.62)$ y $38,84(\mathrm{DT}=7,36)$ para mujeres, mostrando estereotipos negativos en ambos casos.

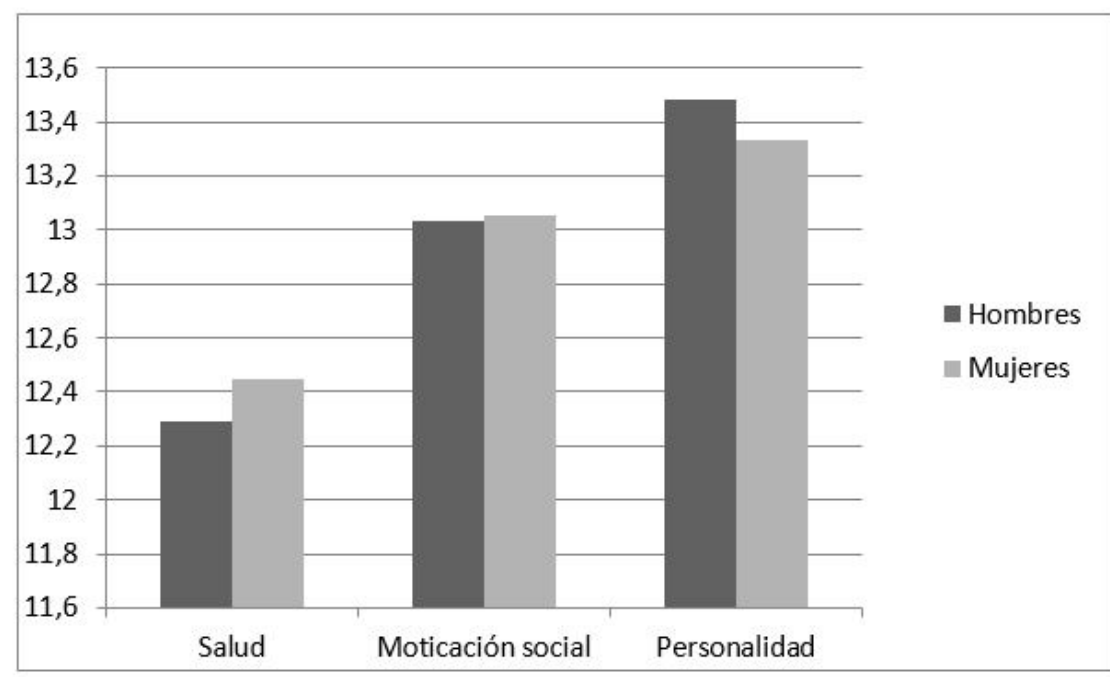

FIGURA 2

Diferencias en las subescalas del CENVE

Fuente: Elaboración propia

\section{Discusión}

El trabajo presentado forma parte de un proyecto en el cual se estudian los estereotipos sobre la vejez en distintos programas universitarios para posteriormente desarrollar programas de intervención dirigidos a promover un mejor conocimiento de los procesos de envejecimiento humano. Concretamente, haciendo referencia al ámbito educativo, las revisiones de estudios sobre el tema anteriormente expuesto suponen una justificación para invitar a la reflexión a los centros educativos sobre cómo trabajar la imagen de las personas mayores entre la comunidad educativa.

Los resultados muestran un alumnado de magisterio con estereotipos negativos sobre la vejez. Destacan las puntuaciones más altas en los aspectos relacionados con la personalidad y carácter de las personas mayores. Esto implica la creencia de que las personas mayores vuelven a ser como niños, y que la edad implica mayor rigidez mental y problemas de regulación emocional, pero estas creencias son consideradas desfasadas y sesgadas. Contra este prejuicio de infantilismo de la vejez, hay que utilizar el término de adultos mayores.

En la dimensión de motivación social, se mantienen también los estereotipos de que los intereses vitales disminuyen con el envejecimiento.

Llama la atención que los participantes del estudio no muestran estereotipos negativos en la dimensión de salud. Parece que el futuro personal docente tiene la creencia de que los 65 años no marcan de manera homogénea el comienzo de las enfermedades y dependencia. También es de señalar que, los nuevos estilos de envejecimiento activo o positivo ofrecen una imagen más saludable del mayor.

Tal y como se observa en los resultados, tanto hombres como mujeres muestran estereotipos de edad, sin obtenerse diferencias significativas entre los géneros, siendo estos resultados similares a los obtenidos en otros estudios (Aristizábal, Morales, Camila y Marcela, 2009). 
La importancia de las personas mayores en la sociedad actual queda reconocida no sólo en los datos socio demográficos, sino a través de los nuevos roles que desempeñan los mayores fruto de estilos de envejecimiento más activos y saludables.

El papel que tienen abuelos y abuelas asumiendo el cuidado de los nietos, y su relación con los centros educativos, suponen una realidad visible en las puertas de muchos colegios. También son una fuente de enseñanza, participando de manera activa en los procesos de socialización de sus nietos. Además, este contacto invita a los niños a reflexionar sobre el curso de la vida y de la muerte, y les ayuda a tomar conciencia del proceso de la vida humana, del pasado y del presente, conceptos que suelen trabajarse en la etapa de la educación primaria. La relación de los abuelos y los nietos es muy significativa para el desarrollo personal, social y afectivo de ambos (Triadó y Villar, 2000).

El aumento del número de personas mayores y los nuevos roles que desempeñan en la sociedad están presente en las escuelas de dos maneras distintas; por un lado, el rol de los abuelos y su relación con los contextos educativos, como acompañantes a la escuela, ayuda en la ejecución de los deberes, como apoyo de muy diferente índole en la infancia de sus nietos, entre otros. Y, por otro lado, con el envejecimiento del profesorado, que implica nuevas formas de relación intergeneracional entre jóvenes y viejos docentes.

La presencia de estereotipos sobre la vejez entre los futuros docentes evidencia la necesidad de plantear pautas de actuación en contextos educativos para disminuir esta visión negativa (Gallardo et ál., 2016).

Es necesario tener en cuenta que los estereotipos son adquiridos por los niños a partir de la observación, cuando se encuentran situaciones de discapacidad o desamparo en personas mayores. Los niños aprenden de lo que ven, sin necesidad de referencia explícita por parte de los adultos (Levy, 2003). A partir del momento en que los niños interiorizan estos estereotipos, comienza a generar expectativas negativas hacia su propio futuro de envejecimiento (Arias, 2014).

Partimos de la base de que la experiencia social de los docentes también determina los pensamientos que dirigen su quehacer docente, y que estas creencias están expuestas a procesos de cambio. Por lo tanto, una tarea derivada de este trabajo consiste en informar de la importancia que tiene el papel de los educadores y futuros docentes en la imagen que sus alumnos van a desarrollar sobre la última etapa de la vida y sobre el grupo de personas mayores. De acuerdo con Nava y Reynoso (2014) para fomentar una cultura de responsabilidad social en el campo educativo es necesario que los educadores hagan una reflexión individual sobre sus fortalezas y debilidades. Esta reflexión puede incluir también sus creencias y conocimientos sobre las diferentes etapas de la vida.

Estos estereotipos se pueden cambiar a través de las vivencias personales. Uno de los elementos que ha recibido más atención en la investigación en el campo de la educación y la psicología para mejorar la imagen de los mayores y cambiar actitudes edadistas son los programas intergeneracionales (Aristizábal et ál., 2009).

Los programas intergeneracionales son vehículos para el intercambio de recursos y aprendizajes entre las generaciones mayores y las más jóvenes con el fin de conseguir beneficios individuales y sociales (Sánchez y Díaz, 2005). Se basan en la noción de que existe una sinergia entre el niño y la persona mayor y de que esta conexión es oportuna y natural y se basa en necesidades recíprocas. Este tipo de programas contribuyen también al envejecimiento activo, mejoran la identidad de las personas, la transmisión de los aprendizajes, expresan nuevos vínculos sociales y propician el diálogo compartido (Tonda, 2011). En otras palabras, las personas mayores son un referente de experiencias, ideas, costumbres y sabiduría para la sociedad, que no puede ser desaprovechado desde el sistema educativo.

Las actividades intergeneracionales son actividades que generan cooperación, intercambio y desarrollo entre personas de diferentes generaciones. Son de muy diferente tipo, educativas, asistenciales, o de voluntariado, pero todas ellas suponen un encuentro entre personas de diferentes grupos de edad, con beneficios para todas ellas.

Aprender cómo son los otros a través de la interacción directa, es uno de los beneficios más destacables de este tipo de programas. 
La mejor forma de romper con los estereotipos negativos y el edadismo es a través de la educación. Una intervención adecuada desde la escuela puede modificar las ideas de los niños en edades tempranas de que es envejecer, contemplando a las personas mayores como personas activas en la sociedad. Las relaciones intergeneracionales en contextos educativos y con experiencias innovadoras que permitan el acercamiento entre grupos de edad tan distintos. A los mayores el encuentro con los niños les ofrece experiencias enriquecedoras y les devuelve el papel activo y el protagonismo que tienen en la sociedad actual (Pinazo y Kaplan, 2007). Por otro lado, las personas mayores pueden ofrecer aprendizajes significativos dentro del aula, trasmitir valores y cultura a los alumnos y a los docentes. No olvidemos el papel de la persona educadora como facilitador de este tipo de experiencias, pues también el sistema educativo debe favorecer contenidos y mecanismos los cuales faciliten un cambio de actitudes, con diseños e intervenciones que influyan en un mejor conocimiento de las personas mayores.

Como posibles limitaciones del estudio, cabe señalar la baja tasa de respuesta por parte del alumnado de magisterio, lo que puede limitar la generalización de los resultados obtenidos.

A partir de este trabajo, se plantea:

- Seguir estudiando los estereotipos sobre las personas mayores y la vejez en estudiantes y futuros profesionales de otras disciplinas universitarias.

- Informar de la importancia que tiene el papel de los educadores en la eliminación de las actitudes edadistas.

- Diseñar, desarrollar y evaluar un plan de trabajo para prevenir la aparición de estereotipos negativos sobre la vejez en estudiantes de magisterio, a partir de los ejemplos de buenas prácticas de los programas intergeneracionales.

\section{ReFERENCIAS}

Abellán, A. y Pujol, A. (2016). Un perfil de las personas mayores en España, 2016. Indicadores estadísticos básicos. Informes de Envejecimiento en red. Recuperado de https://bit.ly/24TxEc5

Almerych, G., Suárez, J., Orellana, N., Belloch, C., Bo, R. y Gastaldo, I. (2005). Diferencias en los conocimientos de los recursos tecnológicos en profesores a partir del género, edad y tipo de centro. Revista Electrónica de Investigación y Evaluación Educativa, 11(2), 127-146. Recuperado de https://bit.ly/30YZ6sf

Arias, E. (2014). La Imagen social del mayor: una comparación entre alumnos universitariosjóvenes y mayores de CastillaLa Mancha. (Tesis Doctoral). Universidad de Castilla-La Mancha, España.

Aristizábal, N., Morales, A., Salas, B. y Torres, A. (2009). Estereotipos negativos hacia los adultos mayores en estudiantes universitarios. Cuadernos hispanoamericanos de psicologia, 9(1), 35-44.

Baltes, P. B., Staudinger, U. M. y Lindenberger, U. (1999). Lifespan psychology: Theory and application to intellectual functioning. Annual review of psychology, 50(1), 471-507. doi: https://doi.org/10.1146/annurev.psych.50.1.471

Blanca, M., Sánchez, C. y Trianes, M. (2005). Cuestionario de evaluación de estereotipos negativos hacia la vejez. Revista multidisciplinar de gerontología, 15(4), 212-221

Bustillos, A. y Fernández, R. (2013). Attributions of competence mediate the behaviors of caregivers and older adults. GeroPsych: The Journal of Gerontopsychology and Geriatric Psychiatry, 26, 211. https://doi.org/10.1024/16629647/a000089

Butler, R. (1969). Ageism: anotherform of bigotry. The Gerontololist, 9(4), 243-246. https://doi.org/10.1093/geron t/9.4_Part_1.243

Carbajo, M.C. (2009). Mitos y estereotipos sobre la vejez. Propuesta de una concepción realista y tolerante. Ensayos, Revista de la Facultad de Educación de Albacete, 24, 87-96 
Franco, M., Villarreal, E., Martínez, L. y Galicia, L. (2010). Estereotipos negativos de la vejez en personal de salud de un Hospital de la Ciudad de Querétaro, México. Revista médica de Chile, 138(8), 988-993. doi: https://doi.or $\mathrm{g} / 10.4067 /$ S0034-98872010000800007

Fuentes, C. L. C. (2014). Análisis de la relación entre las actitudes hacia la vejez y el envejecimiento y los índices de bienestar en una muestra de personas mayores. Revista Española de Geriatría y Gerontología, 49(3), 108-114. h ttps://dou.org/10.1016/j.regg.2013.06.001

Gallardo, A., Fernández, C., Sánchez, J. A., Alarcón, D. y Amian, J. (2016). Percepciones de niños y niñas sobre envejecimiento activo y saludable. En J. L. Castejón (Coord.), Psicología y Educación: Presente y Futuro (pp. 878-884). Alicante: ACIPE.

Hsu, C.Y.(2007). Exploring the model of everyday life information behavior of early retiring teachers. (Tesis de Maestría). National Taiwan University, Taipei, Taiwan.

León, S., Correa, G. y Giacaman, R. A. (2015). Negative ageing stereotypes in students and faculty members from three health science schools. Gerodontology, 32(2), 141-148. doi: https://doi.org/10.1111/ger.12065

Levy, B. R. (2003). Mindmatters: Cognitive and physical effects of aging self-stereotypes. Journal of Gerontology, 58B(4), 203-211. doi: https://doi.org/10.1093/geronb/58.4.P203

Levy, B., Ashman, O. y Dror, I. (2000). To be or not to be: The effects of aging stereotypes on the will to live. OMEGAJournal of Death and Dying, 40(3), 409-420. doi: https://doi.org/10.2190/Y2GE-BVYQ-NF0E-83VR

Levy, B., Hausdorff, J.M., Hencke, R. y Wei, J.Y. (2000). Reducing Cardiovascular Stress with Positive Self-Stereotypes of Aging. Journals of Gerontology Series B: Psychological Sciences and Social Sciences, 55(4), 205-213. doi: https: //doi.org/10.1093/geronb/55.4.P205

López, V. B. I. y Basto, T. S. P. (2010). Desde las teorías implícitas a la docencia como práctica reflexiva. Educación y Educadores, 13(2), 275-291. https://doi.org/10.5294/edu.2010.13.2.6

Madrid, D. (2005). La edad del profesorado en los procesos de enseñanza y aprendizaje de la lengua extranjera. En J.L. Martínez, C. Pérez, N. McLaren y L. Quereda, Towards an understanding of the English Language: Past, Present and Future. Studies in Honour of Fernando Serrano (pp. 519-530). Granada: Editorial Universidad de Granada.

Medina, L. (2014). How to access the conceptions and theories that underlie teaching practices? A theoreticalmethodological reflection based on dialogic discourse analysis. Cultura y Educación, 26(3), 603-616. doi: https ://doi.org/10.1080/11356405.2014.965448

Méndez, F.X., Inglés, C.J., Hidalgo, M.D., García, J.M. y Quiles, M.J. (2003). Los miedos en la infancia y la adolescencia: un estudio descriptivo. Revista Electrónica de Motivación y Emoción, 6(13).150-163.

Montorio, I., Izal, M., Sánchez, M. y Losada, A. (2002) Dependencia y autonomía funcional en la vejez. La profecía que se autocumple. Revista Multidisciplinar de Gerontología, 12(2): 61-71.

Nava, G. N. y Reynoso, J. (2014). Conceptualización y reflexión sobre la práctica educativa en un programa de formación continua para docentes de educación media superior en México. Revista Educación, 39(1), 137-157.

Portal Mayores. (2012). Los mitos y estereotipos asociados a la vejez. 60 y más, 314, 46-51. Recuperado de https://b it.ly/2JQ5VXO

Palmore, E. (1988). The facts on aging: A sort quiz. Gerontologist, 17, 297-313

Palmore, E. (1990). Ageism: Negative and positive. New York: Springer.

Pérez, G. (2004). Calidad de vida en personas mayores. Madrid: Dykinson.

Pinazo, S. y Kaplan, M. (2007). Los beneficios de los programas intergeneracionales. En M. Sánchez (Dir.), Programas intergeneracionales. Hacia una sociedad para todas las edades (pp. 70-101). Barcelona: Fundación "La Caixa".

Posthuma, R. A. y Campion, M. A. (2009). Age stereotypes in the workplace: Common stereotypes, moderates, and future research directions. Journal of Management, 35(1), 158-188. doi: https://doi.org/10.1177/0149206308 318617

Reyes, M. F., Altamar, P., Aguirre, M. y Murillo, D. (2014). Bienestar en personas mayores en situación de pobreza: determinantes y significados. Revista de Psicología, 23(2), 101-115. doi: https://doi.org/10.5354/0719-0581.2 014.36151 
Sánchez, M. y Díaz, P. (2005). Los programas intergeneracionales. En S. Pinazo y M. Sánchez (Eds.), Gerontología. Actualización, innovación y propuestas (pp. 393-426). Madrid: Pearson Educación.

Santamarina, C., López de Miguel, P., López, P. y Mendiguren, V. (2002). Percepciones sociales sobre las personas mayores. Madrid: Ministerio de trabajo y Asuntos sociales.

Santamarina, C. y Marinas, M. (2009). Percepción en niños y adolescentes de las personas mayores. Perfiles y tendencias, 38, 1-52. doi: https://doi.org/10.4067/S0370-41062009000600004

Sanz, R., Mula, J.M. y Moril, R. (2011). La relación abuelos-nietos-escuela: una excusa o una necesidad. XII Congreso Internacional de Teoría de la Educación. Conferencia llevada a cabo en la Universidad de Barcelona, España.

Scholl, J. y Sabat, S. (2008). Stereotypes, stereotype threat and ageing: implications for the understanding and treatment of people with Alzheimer's disease. Ageing \& Society, 28, 103-130.

Tonda, E (2011). Diseño de un proyecto educativo sobre las relaciones intergeneracionales niño-abuelo en la formación de Docentes de Infantil. Aprendizaje a lo largo de la vida, envejecimiento activo y cooperación internacional en los programas universitarios para mayores. Conferencia llevada a cabo en el IV Congreso Iberoamericano de Universidades para Mayores, CIUUM, organizado por la Asociación Estatal de Programas Universitarios para Mayores (AEPUM), Alicante, España.

Triadó, C. y Villar, F (2000). El rol del abuelo: cómo perciben los abuelos las relaciones con sus nietos. Revista Española de Geriatría y Gerontologia, 35(S2), 30-36.

Vega, J. L. y Bueno, B. (1996). Desarrollo adulto y envejecimiento. Madrid: Síntesis.

\section{BY-NC-ND}

